\title{
Use of the AbioCor replacement heart as destination therapy for end-stage heart failure with irreversible pulmonary hypertension
}

\author{
Louis Samuels, MD, ${ }^{\mathrm{a}}$ John Entwistle, MD, ${ }^{\mathrm{b}}$ Elena Holmes, CRNP, ${ }^{\mathrm{a}}$ Jane Fitzpatrick, MD, ${ }^{\mathrm{b}}$ and Andrew Wechsler, MD, \\ Wynnewood and Philadelphia, $\mathrm{Pa}$
}

Because of a potential conflict of interest related to this article on the part of our editors, Dr Robert L. Kormos served as guest editor, assigned reviewers, and made editorial decisions or recommendations leading to its acceptance for publication.


ixed pulmonary hypertension is a contraindication for orthotopic heart transplantation. ${ }^{1}$ The AbioCor artificial heart (Abiomed Inc, Danvers, Mass) was designed as a totally internal replacement system for patients with endstage heart failure who are not eligible for heart transplantation. The purpose of this report is to describe the experience with the AbioCor artificial heart in the setting of a patient with severe biventricular failure and irreversible pulmonary hypertension.

\section{Clinical Summary}

A 51-year-old, 5-foot 9-inch, 170-pound (body surface area 1.9 $\mathrm{m}^{2}$ ) man with idiopathic dilated cardiomyopathy presented with progressive shortness of breath and fatigue. His medical history was significant for emphysema and hepatitis $\mathrm{B}$ and $\mathrm{C}$ viruses. Medications included oral digoxin, hydrazlazine, bumetanide, and fosinopril. Intravenous therapy included dobutamine $10 \mu \mathrm{g} \cdot \mathrm{kg}$. min and milrinone $0.50 \mu \mathrm{g} \cdot \mathrm{kg} \cdot \min$.

Cardiac evaluation showed normal sinus rhythm with left atrial enlargement, 4-chamber cardiac dilatation, severe global biventricular dysfunction, moderate tricuspid and severe mitral regurgitation, and pulmonary hypertension. Left and right heart catheterization showed normal coronary arteries with elevated pulmonary artery $(69 / 37 \mathrm{~mm} \mathrm{Hg})$, central venous $(18 \mathrm{~mm} \mathrm{Hg})$, and pulmonary capillary wedge $(25 \mathrm{~mm} \mathrm{Hg})$ pressures. The thermodilution cardiac output was $3.3 \mathrm{~L} / \mathrm{min}$ with an adjusted cardiac index of $1.7 \mathrm{~L} \cdot \min$

From the Lankenau Hospital, Department of Cardiothoracic Surgery, ${ }^{a}$ Wynnewood, Pa, and Drexel University, Hahnemann University Hospital,

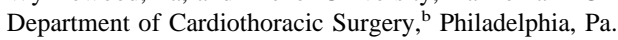

Received for publication July 24, 2003; accepted for publication March 31, 2004.

Address for reprints: Louis E. Samuels, MD, Lankenau Hospital, Department of Cardiothoracic Surgery, MSB Suite 280, 100 Lancaster Avenue, Wynnewood, PA 19096 (E-mail: SamuelsLE@aol.com).

J Thorac Cardiovasc Surg 2004;128:643-5

$0022-5223 / \$ 30.00$

Copyright $\odot 2004$ by The American Association for Thoracic Surgery

doi:10.1016/j.jtcvs.2004.03.052
- $\mathrm{m}^{2}$. The pulmonary vascular resistance was 7.8 Wood units. Pharmacologic maneuvers to reduce the pulmonary vascular resistance with nitroglycerin and nitroprusside were unsuccessful. Stress testing showed a peak oxygen consumption $\left(\mathrm{VO}_{2} \max \right)$ of $8.3 \mathrm{~mL} \cdot \mathrm{kg} \cdot \min$. The estimated ejection fraction was $15 \%$.

Significant laboratory abnormalities included hyponatremia, hypokalemia, and elevated norepinephrine levels. Chest radiography demonstrated hyperinflated lungs and massive cardiomegaly. Pulmonary function studies showed a moderate obstructive pattern on spirometry.

The patient was reviewed by the transplant committee and deemed ineligible on the basis of fixed pulmonary hypertension and comorbidities. He was referred for AbioCor artificial heart trial consideration. The patient agreed to the study and signed the consent form accordingly. The AbioFit was performed and found to be adequate. The AbioScore predicted a 30-day mortality of $75 \%$.

On November 1, 2001, the patient was admitted to Hahnemann University Hospital with decompensated heart failure. The dobutamine infusion was increased to $15 \mu \mathrm{g} \cdot \mathrm{kg} \cdot \mathrm{min}$, and the milrinone was increased to $0.75 \mu \mathrm{g} \cdot \mathrm{kg} \cdot \min$. On November 5 , 2001, the AbioCor device was implanted. Hemodynamics before implantation under general anesthesia were unchanged from those observed in the outpatient clinic (Fig 1). The surgical technique was performed according to protocol, with attachment of the Dacron atrial cuffs to the fibrous skeleton of the atrioventricular valve annuli. The patient was separated from cardiopulmonary bypass after approximately 3.5 hours, with proper pump function and satisfactory hemodynamics (Fig 2). No blood transfusions were required. Transesophageal echocardiography demonstrated no systemic or pulmonary venous inflow obstruction.

The immediate postoperative course was complicated by severe hypoxia and hypercarbia. The chest radiograph showed progressive opacification of both lung fields, left greater than right. Repeat transesophageal echocardiography and bronchoscopy failed to demonstrate a mechanical obstructive problem of the pulmonary veins or bronchi. The right hydraulic pressure (RHP) measurements, a correlate of the pulmonary artery pressure, remained elevated. The patient was supported with extracorporeal membrane oxygenation (ECMO) for 3 days. After withdrawal of ECMO, the patient was extubated on postoperative day 7 with markedly improved arterial blood gas analyses. The AbioCor device flows averaged $6 \mathrm{~L} / \mathrm{min}$. The patient continued to improve clinically and radiographically, allowing for unassisted ambulation and stationary bicycle activities within the first month without supplemental oxygen. He was discharged to a nearby hotel after the second month and was given aspirin, clopidogrel, dipyridamole, and warfarin sodium (Coumadin). Anticoagulation monitoring was determined with standard coagulation studies and thromboelastography. 




Figure 1. Preoperative hemodynamics of patient under general anesthesia with native heart.

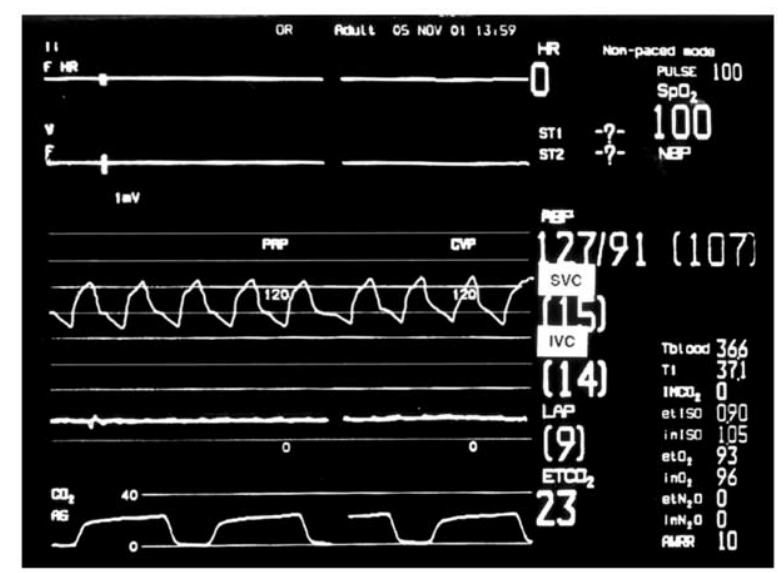

Figure 2. Postoperative hemodynamics of patient under general anesthesia with the AbioCor device (Abiomed Inc, Danvers, Mass).

As an outpatient, the patient was able to participate in many activities, including excursions to church, movies, restaurants, bingo, neighborhood activities, and parties. However, after approximately 3 weeks, his pulmonary condition and oxygen requirements increased, with concomitant elevation of the pump's RHPs and development of prominent interstitial lung markings and pleural effusions on chest radiography. He was readmitted for further investigation.

Operative drainage was required for the relief of serosanguineous pleural effusions. The development of hemoptysis required frequent bronchoscopy and reduction (with eventual cessation) of anticoagulation. His pulmonary condition progressed to ventilator dependence requiring tracheostomy. After several months of support, he was eventually weaned from the ventilator. He was being prepared for discharge when he had a massive stroke on postoperative day 293. The AbioCor device was discontinued, and the patient died. Autopsy findings showed significant emphysema and pulmonary vascular changes consistent with longstanding and severe pulmonary hypertension. There was no obvious evidence of pulmonary emboli on the limited autopsy. Examination of the device showed minor thrombi on the valve leaflets.

\section{Discussion}

The AbioCor replacement heart was approved by the Food and Drug Administration for clinical trial in January 2001. The first clinical implant was performed on July 2, 2001, at the Jewish Hospital, Louisville, Kentucky, with subsequent implants performed at 4 centers in the United States. ${ }^{2}$ Patient selection consists of nontransplant candidates with end-stage biventricular failure on maximal medical therapy whose life expectancy is predicted to be less than 30 days. As such, patients with advanced age, comorbidities with end-organ damage, cancer, and systemic conditions (eg, amyloid) are potential candidates. In addition, patients with elevated and fixed pulmonary hypertension are considered eligible candidates for AbioCor device screening.

The AbioCor is the only device that is a total heart replacement system designed for permanent therapy. ${ }^{3}$ Other systems, all left ventricular assist devices, are being considered for this purpose. ${ }^{4}$ The role of permanent total heart replacement in the setting of fixed pulmonary hypertension, therefore, is novel, because the pump will continue to generate physiologic flow in the milieu of a pathologic pulmonary bed. This unnatural condition may exacerbate underlying pulmonary disease. As demonstrated in this case, the patient had immediate pulmonary complications necessitating ECMO support. The cause was never elucidated, and the condition resolved after 3 days. Theories regarding the cause include a capillary leak type syndrome from the effects of augmented pulmonary blood flow provided by the pump in the setting of a "stiff" pulmonary vascular system. Alternatively, temporary pulmonary venous obstruction caused by the active fill mechanism of the device may have resulted in transient but extreme pulmonary venous congestion. A similar but less dramatic form of this syndrome was observed in another recipient (personal communication from Robert D. Dowling, 2004). In the case presented, the patient's respiratory condition initially improved to a state superior to his preoperative condition at approximately 1 month. His overall health continued to improve during the second month, at which time he was discharged. Shortly thereafter, his pulmonary status gradually deteriorated, requiring rehospitalization and intubation. Again, the cause of this deterioration was unclear. Hemoptysis was an ever-present condition on his readmission and only ceased with discontinuation of anticoagulation and antiplatelet therapy. Attempts to avoid pressure-related causes for the hemoptysis were made by adjusting the pump output. The attempt was to reduce the pump's flow and facilitate a reduction in the RHP, indicating a decrease in the pulmonary artery pressure. A Swan-Ganz catheter (Edwards LifeSciences, Irvine, Calif) was inserted to confirm the accuracy and correlation of the RHP measurements. These maneuvers, and the use of nitric oxide, were unsuccessful. For several weeks during the ventilator support, the RHP remained elevated, and measurements were nearly systemic at times. After cessation of the hemoptysis and aggressive nutritional support, the patient 
was eventually weaned from ventilator support but had a massive stroke at nearly 10 months.

\section{Conclusion}

The AbioCor replacement heart supported the circulation in a patient with end-stage biventricular heart failure with fixed pulmonary hypertension for 293 days. There were no device malfunctions. Physiologic flows permitted activities that exceeded the preoperative state for several months. Pulmonaryrelated complications developed in the immediate postoperative period and resolved. Respiratory failure redeveloped at 2 months and persisted. The unnatural history of fixed pulmonary hypertension in the setting of a total heart replacement system remains an entity of uncertainty. Further studies will be neces- sary to determine whether fixed pulmonary hypertension is an indication or contraindication to destination therapy with a total heart replacement system.

\section{References}

1. Cimato TR, Jessup M. Recipient selection in cardiac transplantation: contraindications and risk factors for mortality. J Heart Lung Transplant. 2002;21:1161-73. Review.

2. Dowling RD, Gray LA Jr, Etoch SW, Laks H, Marelli D, Samuels L, et al. The AbioCor implantable replacement heart. Ann Thorac Surg. 2003;75(6 Suppl):S93-9.

3. Samuels LE, Dowling R. Total artificial heart: destination therapy. Cardiol Clin. 2003;21:115-8.

4. Kukuy EL, Oz MC, Rose EA, Naka Y. Devices as destination therapy. Cardiol Clin. 2003;21:67-73.

\title{
Successful bilateral lung volume reduction in a child with emphysema from bronchiolitis obliterans
}

\author{
Maurizio Mancuso, MD, ${ }^{a}$ Donatella Pacchioni, MD, ${ }^{\mathrm{c}}$ Enrico Ruffini, MD, \\ Antonio Cavallo, MD, ${ }^{a}$ Pierluigi Filosso, MD, ${ }^{a}$ Aurelio Viale, MD, ${ }^{\mathrm{b}}$ Paolo Solidoro, MD, \\ and Elisabetta Bignamini, MD, ${ }^{\mathrm{e}}$ Torino, Italy
}

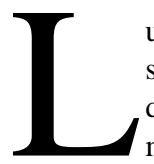
ung hyperinflation occurs not only because of emphysema but also because of small airways diseases with distal air trapping. In infancy bronchiolitis is quite common among airways diseases. We describe the case of a 9-year-old child with end-stage emphysema caused by bronchiolitis and associated chest wall deformity, who was excluded from lung transplantation as a result of severe osteoporosis and who underwent successful bilateral lung volume reduction (LVR) through a median sternotomy.

\section{Clinical Summary}

At the age of 18 months, because of dyspnea and pulmonary infiltrates, the infant underwent an open middle lobe biopsy with evidence of aspecific bronchiolitis obliterans. Furthermore, the patient had a chest wall abnormality consistent with pectus cari-

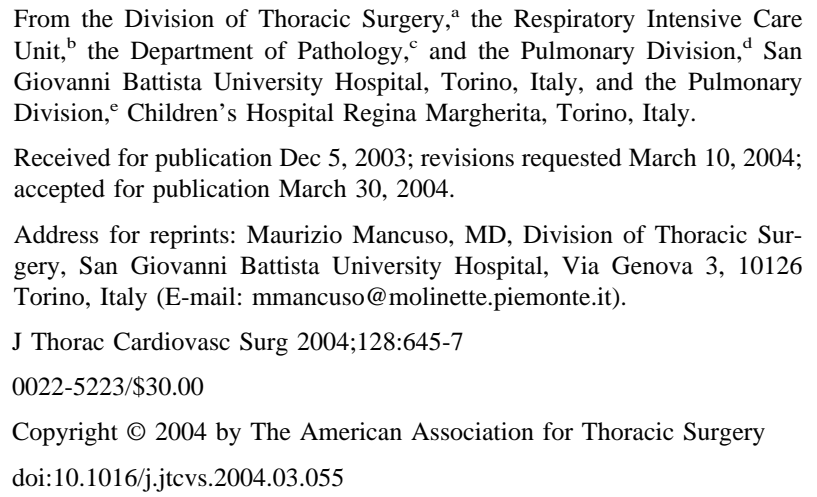

natum and kyphosis (Figure 1). Pulmonary function tests (PFTs) throughout childhood and chest radiographs were consistent with chronic ob-

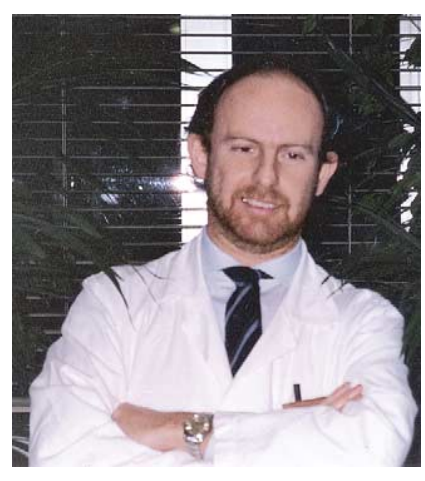

Dr Mancuso structive pulmonary disease. $\alpha 1$-Antytripsin levels and sweat test results were normal.

At the age of 7 years, he worsened, with severe exertional dyspnea necessitating frequent hospitalizations. Prednisone was augmented to $1 \mathrm{mg} / \mathrm{kg}$. The patient was referred to a lung transplantation program abroad because of his age. Meanwhile, his osteoporosis became symptomatic, with spontaneous fracture of the right clavicle, and the child was again referred to us. Recalcification therapy was then started, along with tapering of prednisone to $0.3 \mathrm{mg} / \mathrm{kg}$. The bone disease improved, but a further worsening of respiratory function occurred.

He quit going to school and was very disabled at home in New York Heart Association class III. Forced expiratory volume in 1 second $\left(\mathrm{FEV}_{1}\right)$ decreased to $26 \%$, total lung capacity (TLC) was $132 \%$, residual volume (RV) was $310 \%$, and RV/TLC was $226 \%$ of predicted value. The diffusing capacity of the lung for carbon monoxide was $75 \%$ of the normal values, $\mathrm{PaO}_{2}$ was $61 \mathrm{~mm} \mathrm{Hg}$, and $\mathrm{CO}_{2}$ was normal. During the 6-minute walking test, he walked $440 \mathrm{~m}$, and on the treadmill test, he completed the sixth level with $4 \mathrm{~L}$ of oxygen, with a dyspnea Borg index of 14. A high-resolution computed tomograph showed lung hyperinflation and luscency, a transmediastinal hernia of the right upper lobe, and medium-sized bullae in the left lower lobe.

A Q scan showed $62 \%$ perfusion on the right lung and $38 \%$ on the left. Very low nuclear uptake was observed in the left lower lobe $(6 \%)$ and in the right upper lobe $(13 \%)$. 